\title{
A HEURISTIC METHOD FOR THE DETERMINATION OF A HAMILTONIAN CIRCUIT IN A GRAPH
}

\author{
SUDHANGSHU B. KARMAKAR ${ }^{1}$
}

(Received 27 August 1985; revised 31 December 1985)

\begin{abstract}
A heuristic methodology for the identification of a circuit passing through all the vertices only once in a graph is presented. The procedure is based upon defining a normal form of a matrix and then transforming the adjacency matrix into its normal form. For a class of graphs known to be Hamiltonian, it is conjectured that this methodology will identify circuits in a small number of steps and in many cases merely by observation.
\end{abstract}

\section{Introduction}

A circuit in a connected graph is said to be Hamiltonian if it includes every vertex once. Hence a Hamiltonian circuit in a graph of $n$ vertices consists exactly of $n$ edges. The problem of finding an efficient algorithm for identifying a Hamiltonian circuit in a graph has offered an interesting challenge to the applied mathematicians and graph theorists since it was first proposed by Sir William Rowan Hamilton in 1895. The basic unvolved problem of determining which graphs are Hamiltonian has led to the investigation of many related problems, and consequently, to the development of what might generally be termed, "Hamiltonian theory". Since it has been shown $[3,7]$ that the general problem for finding a Hamiltonian circuit in a graph is $N P$-complete, attention has been directed to the development of efficient algorithms for some special but useful cases. Sufficient conditions for the existence of a Hamiltonian circuit have been obtained in terms of the degree sequence of a graph. Polynomial-time algorithms have been given for finding a Hamiltonian cycle in a graph satisfying such

\footnotetext{
I Bell Communications Research, Piscataway, NJ 08854, USA.

(c) Copyright Australian Mathematical Society 1987, Serial-fee code 0334-2700/87
} 
sufficient conditions. In this paper some known results regarding Hamiltonian circuits are reviewed and a heuristic algorithm is presented to determine a Hamiltonian circuit in a graph.

\section{Preliminaries}

Standard graph theory terminology $[1,2,5]$ is used. Formally, a graph $G=$ $(V, E)$ consists of a finite nonempty set of vertices $V$ and a set of edges $E$. If the edges are ordered pairs $(u, v)$ of vertices, then the graph is said to be directed. If the edges are unordered pairs of distinct vertices also denoted by $(u, v)$, then the graph is said to be undirected. A directed graph is also called a digraph. The order of a graph $n$ denotes the number of vertices of $G: n=|V|$.

An edge $u v$ is said to join the vertices $u$ and $v$ and is denoted by $u v$. If $u v \in E$ then $u$ and $v$ are adjacent and the vertices $u$ and $v$ are incident with edge $u v$.

The size of a graph $e$ is the number of edges i.e. $e=|E|$. A graph of order $n$ and size $\left(\begin{array}{l}n \\ 2\end{array}\right)$ is called a complete graph and is denoted by $K_{n}$. In $K_{n}$ every two vertices are adjacent.

Simple graph. A graph is simple if there is no loop of length one at any one of the vertices.

A digraph is strict if it has no loop and no two edges with the same vertices have the same orientation.

A graph is bipartite if the vertex set $V$ can be partitioned into two subsets $V_{1}$ and $V_{2}$ such that every edge of $G$ joins $V_{1}$ with $V_{2}$.

The degree of a vertex $v$ in a graph $G$ is the number of edges of $G$ incident with $v$. In a digraph the outdegree $\operatorname{od}(v)$ of a vertex $v$ is the number of vertices adjacent to it. A walk of a graph $G$ is an alternating sequence of vertices and edges $v_{1} E_{1} v_{2} E_{2} \cdots E_{k} v_{k}$, beginning and ending with points in which each edge is incident with the two points immediately preceding and following it. This walk joins $v_{1}$ and $v_{k}$ and may also be denoted by $v_{1} v_{2} \cdots v_{k}$; it is sometimes called a $v_{1} v_{k}$ walk. The walk is closed if $v_{1}=v_{k}$ and is open otherwise. A path is a walk where all the vertices are distinct. A circuit or a cycle is a walk $v_{1} v_{2} \cdots v_{k}$ where all the vertices are distinct except for $v_{1}=v_{k}$. The length of a walk is the number of occurrences of edges in it. A digraph is diconnected if for any two vertices $u$ and $v$ there is a directed path from $u$ to $v$. A graph is connected if for every pair $(u, v)$ of distinct vertices there is a path from $u$ to $v$.

The $k$-closure $C_{k}(G)$ of a simple graph $G$ of order $n$ is the graph obtained from $G$ by recursively joining pairs of nonadjacent vertices with degree at least $k$.

The adjacency matrix $A=\left[a_{i j}\right]$ of $G$ is the $n \times n$ matrix given by

$$
a_{i j}= \begin{cases}1 & \text { if } v_{i}, v_{j} \in E \\ 0 & \text { otherwise }\end{cases}
$$




\section{Sufficient conditions for a Hamiltonian graph}

Even though no elegant characterization of a Hamiltonian graph has yet been found there have been several sufficient conditions established for a graph to be Hamiltonian. Chronologically, Dirac, Ore, Posa, Bondy and Chvatal have determined such conditions in terms of the degrees of the vertices of a graph, with each successive result strengthening those preceding it [8]. A graph $G$ of order $n \geqslant 3$ with degree sequence $d_{1} \leqslant d_{2} \cdots \leqslant d_{n}$ is Hamiltonian if:

(i) (Dirac) $1 \leqslant k \leqslant n \Rightarrow d_{k} \geqslant n / 2$

(ii) (Ore) $u v \notin E \Rightarrow \operatorname{deg} u+\operatorname{deg} v \geqslant n$

(iii) (Posa) $1 \leqslant k<n / 2 \Rightarrow d_{k}>k$

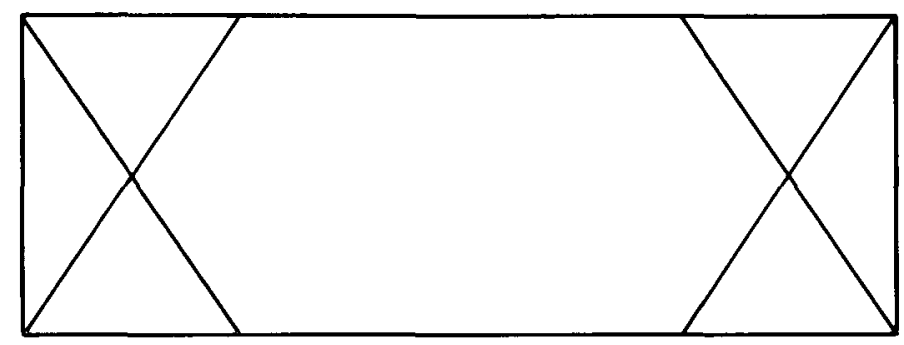

Figure 1a. A Hamiltonian graph which does not satisfy the sufficient conditions of Posa, Ore, Dirac.

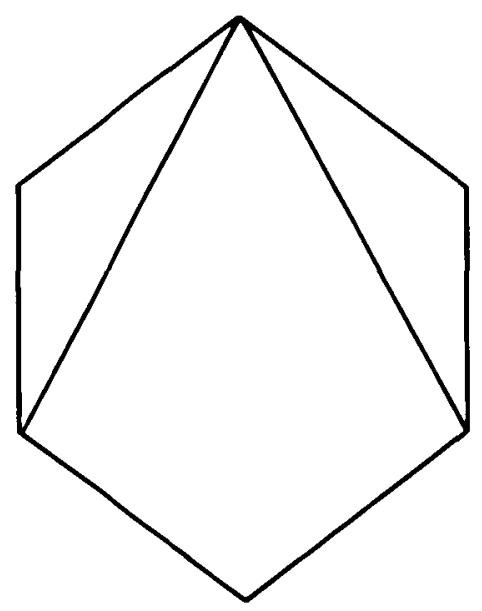

Figure 1b. A Hamiltonian graph which does not satisfy the condition of Las Vergnas.

Figure 1. Hamiltonian graphs which do not satisfy sufficient conditions. 
(iv) (Bondy) $j<k, d_{j} \leqslant j, d_{k} \leqslant k-1 \Rightarrow d_{j}+d_{k} \geqslant n$

(v) (Chvatal) $d_{k} \leqslant k<n / 2 \Rightarrow d_{n-k} \geqslant n-k$

(vi) (Las Vergnas) A graph $G$ of order $n \geqslant 3$ is Hamiltonian if the vertices can be labeled $v_{1}, v_{2}, \ldots, v_{k}$ so that

$$
\left.\begin{array}{r}
j<k, k \geqslant n-j, v_{j} v_{k} \notin E \\
\operatorname{deg} v_{\jmath} \leqslant j, \operatorname{deg} v_{k} \leqslant k-1
\end{array}\right\} \Rightarrow \operatorname{deg} v_{\jmath}+\operatorname{deg} v_{k} \geqslant n
$$

(vii) If $G$ is a graph of order $n$ and the $n$ closure $C_{n}(G)$ is complete, then $G$ is Hamiltonian. It can be shown [8] that conditions (i)-(vi) guarantee that $C_{n}(G)$ is complete. However, (vii) is strictly stronger than all of these conditions.

(vii) (Meyniel) In a strict diconnected digraph $G$, if $u v \notin E$ and $\operatorname{deg}(u)+$ $\operatorname{deg}(v) \geqslant 2 n-1$ for all $u, v \in V$ then $G$ contains a direct Hamiltonian circuit.

The above conditions are sufficient but not necessary for the existence of a Hamiltonian circuit in a graph. For example the graph shown in Figure 1a is Hamiltonian yet it does not satisfy the conditions (i), (ii) and (iii). The graph shown in Figure 1b is Hamiltonian by (vii) but it does not satisfy the condition (vi).

\section{Conditions in terms of edges}

Related results involving the degree of the vertices of a graph are those involving the number of edges of the graph. Ore [9] showed that $G$ is Hamiltonian if $n=|V| \geqslant 3$ and $|E| \geqslant\left(n^{2}-3 n+6\right) / 2$. Another result due to Posa [10] states that there exists a constant $c$ such that the probability that a random graph with $n$ vertices and $c n \log n$ edges is Hamiltonian tends to 1 as $n \rightarrow \infty$.

\section{Necessary condition}

Since a Hamiltonian circuit must pass through each of the vertices, a simple necessary condition can be obtained [6] in terms of the power of the adjacency matrix of $G$ : Let $A^{n}=\left[a_{i j}^{(n)}\right]$. Since $a_{i j}^{(n)}>0$ implies there exists a walk of length $n$ between $v_{i}$ and $v_{j}$, the necessary condition for the existence of a circuit of length $n$ at each of the vertices is that $a_{i i}^{(n)}>0$ for all $i \leqslant n$. 


\section{Heuristic method}

A heuristic may be viewed as a less-than-perfect method [4] which usually finds a solution. In this paper the word heuristic is used in the sense of a method for guiding discovery or improving problem solving. In order to develop the method to determine a Hamiltonian circuit in $G$ by heuristic, the following definitions will be essential.

Normal form of a matrix: Let $n$ be the dimension of the adjacency matrix $A$ and $h$ be an integer such that $1 \leqslant h \leqslant n-1$. For all $i, 1 \leqslant i \leqslant n$ if $A\left(i, \bmod _{n}(i\right.$ $+h))=1$ with the modification that $\bmod _{\hat{n}}(n)=n$, then $A$ is said to have a normal form. In this paper the normal form is taken to be of first order i.e. $h=1$.

Normal path of a matrix. The path made up of the off-diagonal elements of a matrix, i.e. made up of $(n-1) a_{i i+1}$ elements such that $1 \leqslant i \leqslant n-1$.

The anchor of a matrix. The element corresponding to $a_{n 1}$.

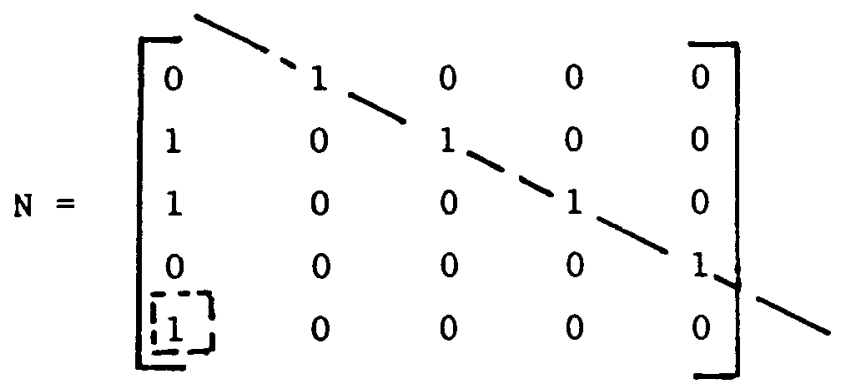

Figure 2a. Matrix $N$ is in normal form.

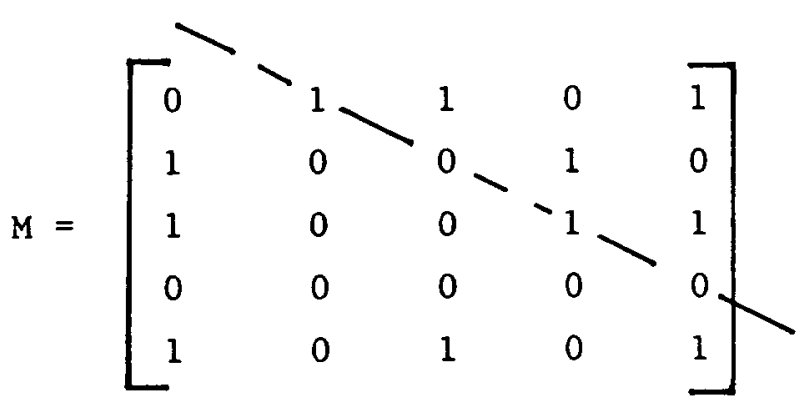

Figure 2b. Matrix $M$ is not in normal form.

Figure 2. Normal form of a matrix. 

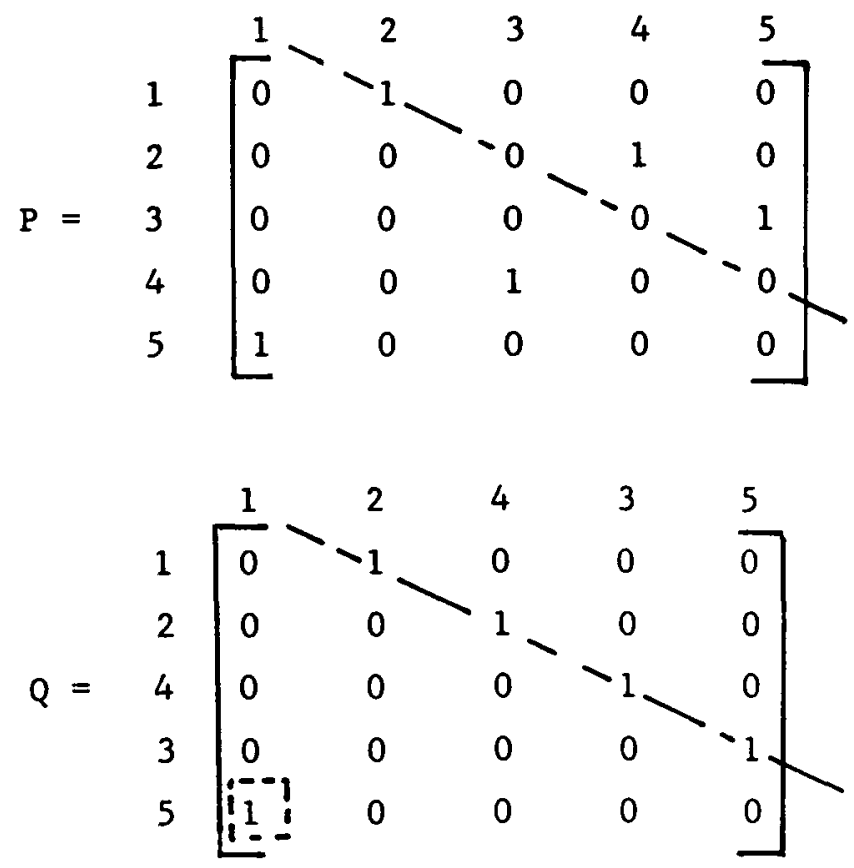

Figure 3a. Matrix $P$ is transformed into matrix $Q$.

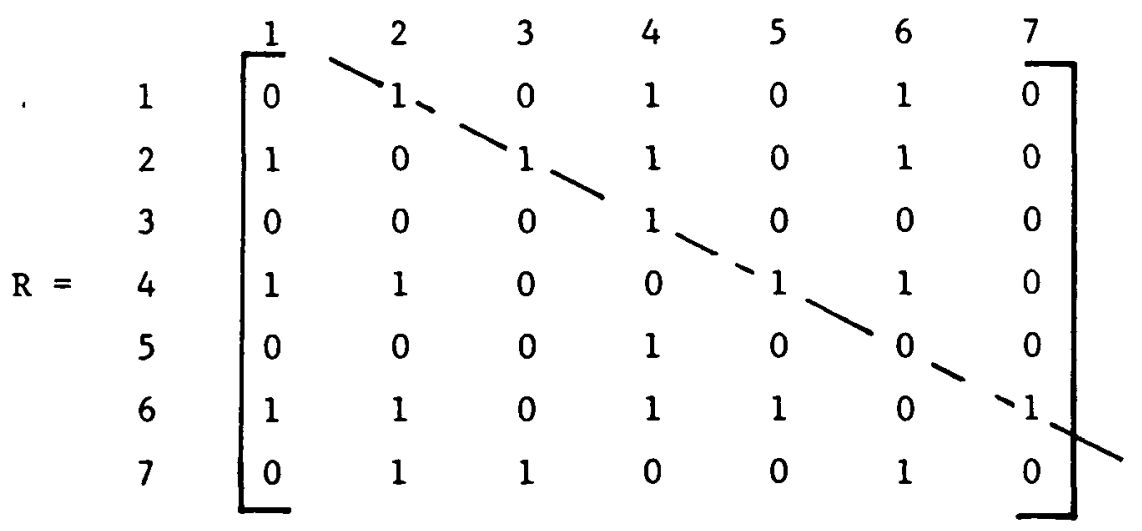

Figure 3b. Matrix $R$ cannot be transformed into normal form.

Figure 3. Transformation into normal form. 
Note in the definitions of normal form, normal path and anchor that the other elements of the matrix are of no consequence. The matrix $N$ shown in Figure $2 \mathrm{a}$ is in normal form while the matrix $M$ shown in Figure $2 \mathrm{~b}$ is not in normal form. If a matrix is not in normal form, attempts can be made to transform it into normal form by interchanging any set of pairs of columns and rows, even though there is no guarantee that a matrix which is initially not in normal form can ultimately be transformed into normal form. For example the matrix $P$ in Figure $3 \mathrm{a}$ is not in normal form. But by first interchanging column 3 and column 4 and then by interchanging corresponding row 3 and $4, P$ is transformed into $Q$ as shown in Figure $3 \mathrm{~b}$ which is in normal form. But no amount of interchanging of pairs of columns and rows will transform the matrix $R$ shown in Figure $3 \mathrm{c}$ into a normal form.

Observation. If a Hamiltonian circuit exists in a graph $G$ then its adjacency matrix must be transformable into normal form, and the row or column headings will identify the circuit. Alternatively, for graphs which are known to be Hamiltonian the corresponding adjacency matrices can always be transormed into normal forms.

\section{A heuristic algorithm to determine a Hamiltonian circuit in a graph}

1. Obtain the adjacency matrix $A=\left[a_{i j}\right], a_{n} \leftarrow 0 i=1,2, \ldots, n$; i.e. make the graph simple.

2. Identify each column and each row by the headings $C_{t}$ and $R_{1}$. Initially each $C_{i}$ and each $R_{i}$ corresponds to each $v_{i}$ and has the value $i, i=1,2, \ldots, n$.

3. Let the set $V_{l}$ contain all the vertices adjacent to the vertex $v_{i} ; v_{\imath} \notin V_{i} \subset V$.

4. If $A$ is in normal form then the Hamiltonian Circuit (HC) is identified as $v_{1}, v_{2} \cdots v_{k}$. Otherwise, go to 5 .

5. Proceed from left to right along the normal path. If a zero element is encountered, set the corresponding column heading as $C_{l}$. Replace this zero element by any nonzero element from the same row corresponding to the column heading $C_{j}$. Note: the replacement of the zero element is done by the interchanging of columns corresponding to $C_{t}$ and $C_{j}$ and rows corresponding to $R_{i}$ and $R_{j}$. While performing this interchanging of rows and columns, there is a possibility that some existing nonzero elements in the normal path may be removed. The index $j$ should be chosen in such a way that the removal of the nonzero elements becomes minimal. This situation corresponds to the "least disturbed" condition of the normal path.

6. If the anchor, i.e. the element corresponding to $R_{n} C_{1}$ is zero, replace it with a nonzero element so that the normal path is least disturbed. 
7. If the matrix is now in normal form go to 8 otherwise go to 5 .

8. Identify the $H C$ by the vertices corresponding to the column headings $C_{1}, C_{2} \cdots C_{n}$.

\section{Illustrative examples}

In order to demonstrate the algorithm, the following two examples are worked out in detail. For the first example it is not known in advance whether the graph contains any Hamiltonian cycle. But for the second example [11] it is known in advance that the graph is Hamiltonian since it satisfies Meyniel's condition.

\section{Example 1.}

Consider the graph of Figure 4.

Steps 1 and 2:

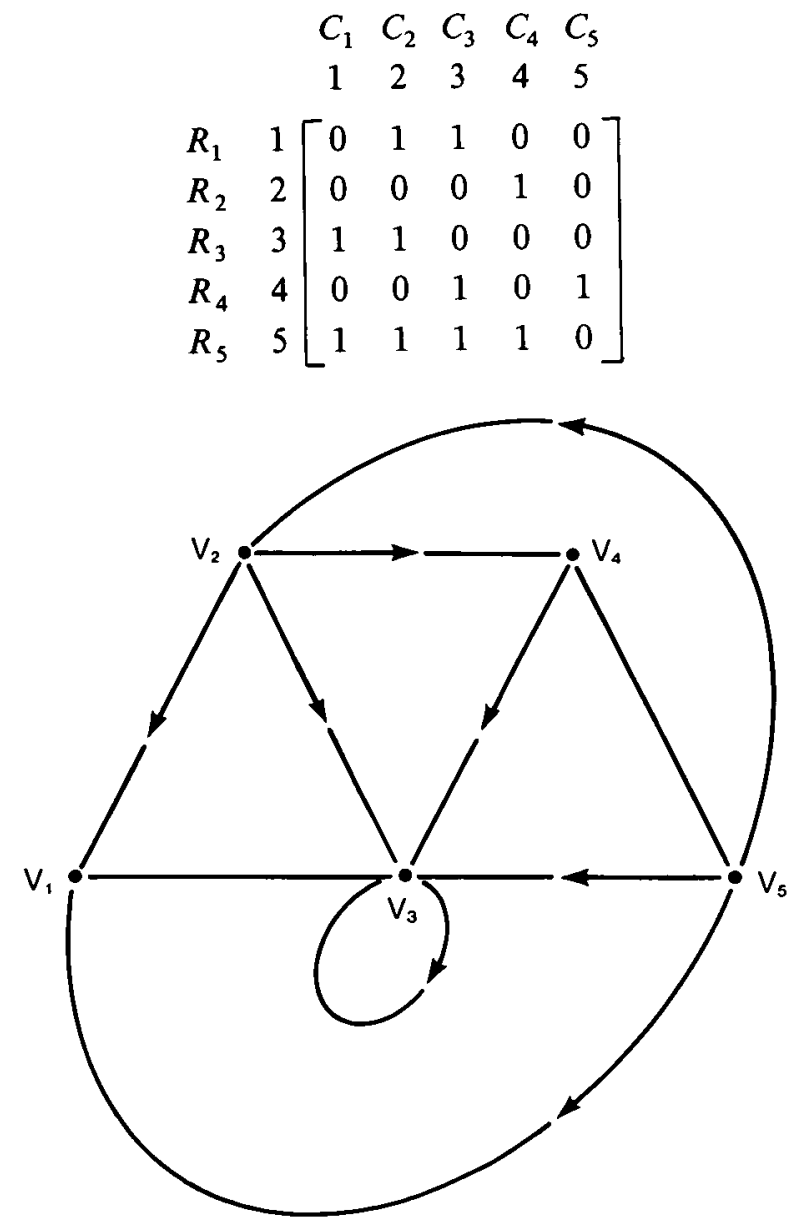

Figure 4. Graph of Example 1. 
Step 3:

$$
\begin{aligned}
& v_{1}: V_{1}=\left\{v_{2}, v_{3}\right\} \\
& v_{2}: V_{2}=\left\{v_{4}\right\} \\
& v_{3}: V_{3}=\left\{v_{1}, v_{2}\right\} \\
& v_{4}: V_{4}=\left\{v_{3}, v_{5}\right\} \\
& v_{5}: V_{5}=\left\{v_{1}, v_{2}, v_{3}, v_{4}\right\}
\end{aligned}
$$

Step 4: $A$ is not in normal form.

Step 5: The first zero element encountered corresponds to $C_{3}$, choose $v_{j} \in V_{2}$, here $j=4$. Interchange columns corresponding to $C_{3}$ and $C_{4}$ and rows corresponding to $R_{3}$ and $R_{4}$. $A$ is thus transformed into $A_{1}$.

$$
\begin{aligned}
& \begin{array}{llllllllllllllll}
C_{1} & C_{2} & C_{3} & C_{4} & C_{5}
\end{array} \\
& \begin{array}{lllll}
1 & 2 & 4 & 3 & 5
\end{array} \\
& A_{1}=\begin{array}{ll}
R_{1} & 1 \\
R_{2} & 2 \\
R_{3} & 4 \\
R_{4} & 3 \\
R_{5} & 5
\end{array}\left[\begin{array}{lllll}
0 & 1 & 0 & 1 & 0 \\
0 & 0 & 1 & 0 & 0 \\
0 & 0 & 0 & 1 & 1 \\
1 & 1 & 0 & 0 & 0 \\
1 & 1 & 1 & 1 & 0
\end{array}\right]
\end{aligned}
$$

The second zero encountered corresponds to $C_{5}$. Since $V_{3}=\left\{v_{1}, v_{2}\right\}$, by replacing columns corresponding to $C_{5}$ and $C_{1}$ the normal path is least disturbed. $A_{1}$ is transformed into $A_{2}$.

$$
\begin{aligned}
& \begin{array}{lllllllllllllllllllll}
C_{1} & C_{2} & C_{3} & C_{4} & C_{5}
\end{array} \\
& \begin{array}{lllll}
5 & 2 & 4 & 3 & 1
\end{array} \\
& A_{2}=\begin{array}{ll}
R_{1} & 5 \\
R_{2} & 2 \\
R_{3} & 4 \\
R_{4} & 3 \\
R_{5} & 1
\end{array}\left[\begin{array}{lllll}
0 & 1 & 1 & 1 & 1 \\
0 & 0 & 1 & 0 & 0 \\
1 & 0 & 0 & 1 & 0 \\
0 & 1 & 0 & 0 & 1 \\
0 & 1 & 0 & 1 & 0
\end{array}\right]
\end{aligned}
$$

The normal path is now made up of 5-1 $=4$ nonzero elements.

Step 6: The anchor corresponding to $R_{5} C_{1}=0$. Since $V_{1}=\left\{v_{2}, v_{3}\right\}$ and the objective is to disturb the normal path the least, interchange columns corresponding to $C_{1}$ and $C_{4}$ and rows corresponding to $R_{1}$ and $R_{4} . A_{2}$ is now transformed into $A_{3}$. 


$$
\begin{array}{rlllll}
C_{1} & C_{2} & C_{3} & C_{4} & C_{5} \\
3 & 2 & 4 & 5 & 1 \\
A_{3}= & R_{1} & 3 \\
R_{2} & 2 \\
R_{3} & 4 \\
R_{4} & 5 \\
R_{5} & 1
\end{array}\left[\begin{array}{llllll}
0 & 1 & 0 & 0 & 1 \\
0 & 0 & 1 & 0 & 0 \\
1 & 0 & 0 & 1 & 0 \\
1 & 1 & 1 & 0 & 1 \\
1 & 1 & 0 & 0 & 0
\end{array}\right]
$$

Step 7: The matrix $A_{3}$ is now in normal form.

Step 8: A Hamiltonian circuit is obtained as follows:

$$
v_{3} v_{2} v_{4} v_{5} v_{1} v_{3}
$$

Example 2: Consider the graph in Figure 5. Its adjacency matrix $A$ is shown below.

$$
\left.A=\frac{1}{2}+\begin{array}{lllllllllll}
1 & 2 & 3 & 4 & 5 & 6 & 7 & 8 & 9 & 10 & 11 \\
3 \\
4 \\
5 \\
6 \\
7 \\
8 \\
9 \\
10 & 1 & 1 & 1 & 0 & 0 & 0 & 1 & 1 & 0 & 1 \\
0 & 0 & 1 & 0 & 0 & 1 & 1 & 1 & 0 & 1 & 1 \\
0 & 0 & 0 & 1 & 1 & 0 & 0 & 0 & 0 & 1 & 1 \\
1 & 1 & 1 & 0 & 1 & 0 & 1 & 1 & 0 & 0 & 1 \\
0 & 1 & 1 & 1 & 0 & 1 & 0 & 0 & 0 & 0 & 1 \\
0 & 0 & 1 & 0 & 1 & 0 & 1 & 1 & 0 & 0 & 1 \\
1 & 1 & 1 & 1 & 0 & 1 & 0 & 1 & 0 & 0 & 1 \\
1 & 1 & 1 & 0 & 0 & 0 & 1 & 0 & 0 & 0 & 1 \\
1 & 0 & 0 & 0 & 1 & 1 & 1 & 1 & 0 & 1 & 1 \\
1 & 1 & 0 & 0 & 1 & 1 & 0 & 0 & 1 & 0 & 1 \\
0 & 0 & 0 & 0 & 0 & 0 & 0 & 0 & 1 & 1 & 0
\end{array}\right]
$$

$A$ is almost in normal form except in column 9 , and in the anchor position. Since $v_{11} \in V_{8}, v_{11}$ is a potential candidate to be brought into the normal path. By interchanging the columns 9 and 11 and the corresponding rows 9 and $11, A$ is transformed into $A_{1}$ which is in the normal form.

$$
\begin{aligned}
& \begin{array}{lllllllll}
2 & 3 & 4 & 5 & 6 & 7 & 8 & 11 & 10
\end{array} \\
& \begin{array}{l}
1 \\
2
\end{array}\left[\begin{array}{lllllllllll}
0 & 1 & 1 & 1 & 0 & 0 & 0 & 1 & 1 & 0 & 1 \\
0 & 0 & 1 & 0 & 0 & 1 & 1 & 1 & 1 & 1 & 0
\end{array}\right] \\
& 30 \begin{array}{lllllllllll}
3 & 0 & 0 & 1 & 1 & 0 & 0 & 0 & 1 & 1 & 0
\end{array} \\
& \begin{array}{llllllllllll}
4 & 1 & 1 & 1 & 0 & 1 & 0 & 1 & 1 & 1 & 0 & 0
\end{array} \\
& \begin{array}{l|lllllllllll}
A_{1}=5 & 0 & 1 & 1 & 1 & 0 & 1 & 0 & 0 & 1 & 0 & 0 \\
6 & 0 & 0 & 1 & 0 & 1 & 0 & 1 & 1 & 1 & 0 & 0
\end{array} \\
& \begin{array}{llllllllllll}
7 & 1 & 1 & 1 & 1 & 0 & 1 & 0 & 1 & 1 & 0 & 0
\end{array} \\
& \begin{array}{llllllllllll}
8 & 1 & 1 & 1 & 0 & 0 & 0 & 1 & 0 & 1 & 0 & 0
\end{array}
\end{aligned}
$$

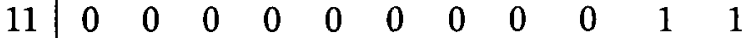

$$
\begin{aligned}
& \begin{array}{c}
10 \\
9
\end{array}\left[\begin{array}{lllllllllll}
1 & 1 & 0 & 0 & 1 & 1 & 0 & 0 & 1 & 0 & 1 \\
1 & 0 & 0 & 0 & 1 & 1 & 1 & 1 & 1 & 1 & 0
\end{array}\right]
\end{aligned}
$$

A Hamiltonian circuit is identified as follows:

$$
v_{1} v_{2} v_{3} v_{4} v_{5} v_{6} v_{7} v_{8} v_{11} v_{10} v_{9} v_{1}
$$




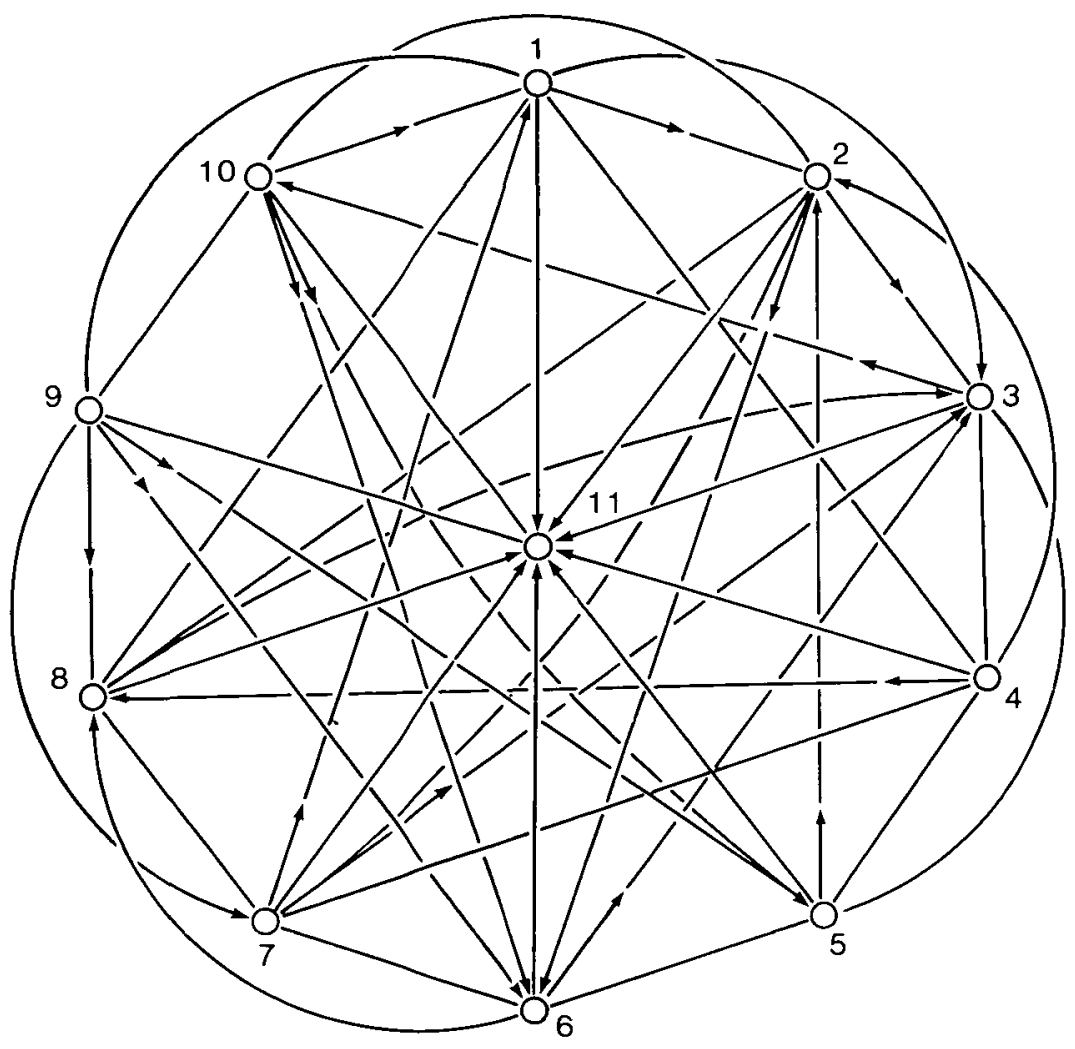

Figure 5. Graph of Example 2.

\section{Concluding remarks}

In conclusion several characteristic features of the proposed algorithm are noted. The algorithm is applicable for both directed and undirected graphs. The essential element of the algorithm is first to define a normal form of the adjacency matrix and then try to transform the matrix into its normal form. The normal form is obtained by interchanging pairs of columns and corresponding pairs of rows. Thus the most important aspect of the algorithm is to determine the best possible candidate to be interchanged with. Otherwise, there is a potential risk that these interchanges will result in the method cycling in a loop. In selecting the "best candidate" some looking-ahead aspect should be introduced so that the "least disturbance" here does not cause a major disturbance later. However, there is no efficient algorithm known to determine the best candidate. If the adjacency matrix of a Hamiltonian graph is crowded with 1's, then it is quite likely that it 
can be transformed into a normal form in some smaller number of steps and in many cases merely by observation. The adjacency matrices of the graphs which satisfy the sufficient conditions as stated by Ore, Dirac and Meyniel are very dense with 1's. Thus it is postulated that for the above situations, the complexity of the determination of a Hamiltonian Circuit is $0(n)$.

\section{References}

[1] A. V. Aho, J. E. Hopcroft, and J. D. Ullman, The design and analysis of computer algorithms (Addison-Wesley, 1974).

[2] B. Bollobas, Graph Theory (Springer-Verlag, 1979).

[3] M. R. Gary, D. S. Johnson and R. E. Taran, "The planar Hamiltonian circuit problem is NP complete", SIAM J. Comput. 5 (1976), 704-714.

[4] R. Groner, H. Groner and W. F. Bischof, Methods of Heuristics (Lawrence Erlbaum Asso. 1983).

[5] F. Harary, Graph Theory (Addison-Wesley, 1972).

[6] S. B. Karmakar, "Detection of Hamiltonian circuits in a directed graph", J. Austral. Math. Soc. Ser. B 24 (1982), 234-242.

[7] M. S. Krishnamoorthy, "An NP hard problem in bipartite graphs", SIGACT New 7 (1975), $1-2 \mathrm{C}$.

[8] L. Lesniak-Foster, "Some recent results in Hamiltonian graphs", J. Graph Theory 1 (1977), 27-36.

[9] O. Ore, “Arc coverings of graphs", Ann. Mat. Pura Appl. 55 (1961), 315-322.

[10] L. Posa, "Hamiltonian circuits in random graphs", Discrete Math. 14 (1976), 359-364.

[11] K. Takamizawa, T. Niskizehi and N. Saito, "An $O\left(p^{3}\right)$ algorithm for finding Hamiltonian cycles in certain digraphs", J. Inform. Process, 3 (1980), 68-72. 